\title{
Detection of a population gradient in the Sagittarius stream
}

\author{
M. Bellazzini ${ }^{1}$, H. J. Newberg ${ }^{2}$, M. Correnti ${ }^{1}$, F. R. Ferraro ${ }^{3}$, and L. Monaco ${ }^{4, \star}$ \\ 1 INAF - Osservatorio Astronomico di Bologna, Via Ranzani 1, 20127 Bologna, Italy \\ e-mail: michele.bellazzini@oabo.inaf.it \\ 2 Department of Physics and Astronomy, Rensselaer Polytechnic Institute, Troy, NY 12180, USA \\ 3 Dipartimento di Astronomia, Università di Bologna, Via Ranzani 1, 20127 Bologna, Italy \\ ${ }^{4}$ ESO - European Southern Observatory, Alonso de Cordova 3107, Santiago 19, Chile
}

Received 10 July 2006 / Accepted 16 August 2006

\section{ABSTRACT}

\begin{abstract}
We present a quantitative comparison between the Horizontal Branch morphology in the core of the Sagittarius dwarf spheroidal galaxy (Sgr) and in a wide field sampling a portion of its tidal stream (Sgr Stream), located tens of kpc away from the center of the parent galaxy. We find that the Blue Horizontal Branch (BHB) stars in that part of the Stream are five times more abundant than in the Sgr core, relative to Red Clump stars. The difference in the ratio of BHB to RC stars between the two fields is significant at the $\gtrsim 4.8 \sigma$ level. This indicates that the old and metal-poor population of Sgr was preferentially stripped from the galaxy in past periGalactic passages with respect to the intermediate-age metal rich population that presently dominates the bound core of Sgr, probably due to a strong radial gradient that was settled within the galaxy before its disruption. The technique adopted in the present study allows to trace population gradients along the whole extension of the Stream.
\end{abstract}

Key words. galaxies: dwarf - galaxies: evolution - stars: horizontal-branch

\section{Introduction}

The Sagittarius dwarf spheroidal galaxy (Sgr dSph; Ibata et al. 1994) provides an excellent case for the study of the process of tidal disruption and accretion of a dwarf satellite into a large galaxy. Its huge tidal tails form a coherent and dynamically cold filamentary structure (hereafter Sgr Stream) extending for tens of kpc from the parent galaxy that has been probed with many different tracers (see, among the others, Ibata et al. 2001; Newberg et al. 2002, hereafter N02; Majewski et al. 2003, hereafter M03; Martínez-Delgado et al. 2004, hereafter MD04; Vivas et al. 2005; Belokurov et al. 2006, and references therein).

It is quite clear that the progenitor of the presently observed Sgr remnant was a quite remarkable galaxy, possibly similar to the LMC (Monaco et al. 2005, hereafter M05). While the stellar content of the bound core of the galaxy is dominated by an intermediate-old metal-rich population $([\mathrm{M} / \mathrm{H}] \sim-0.5$, M05, Bellazzini et al. 2006), the globular clusters possibly associated with the Stream are predominantly old and metal-poor (Bellazzini et al. 2003; see also Belokurov et al. 2006). M03 and MD04 provided the first indications of the possible presence of a metallicity gradient along the Stream, suggesting that the stars lost in previous peri-Galactic passages, were, on average, more metal poor than those recently lost or still bound to the main body. Chou et al. (2006, hereafter C06), studying the chemical abundances of relatively nearby Stream stars, have shown that the metallicity distribution within the Stream is significantly different from that of the main body of Sgr, the former being skewed toward lower metallicities. These results strongly suggest that the stellar content of the Sgr progenitor could have

* Based on observations collected at the European Southern Observatory, Chile (Programme 71.D-0222A). been quite different from the present-day remnant, and indicates that it may be possible to trace the change of stellar content along the Stream (see C06).

The Horizontal Branch (HB) morphology is a very powerful tool to study population gradients within galaxies (see Harbeck et al. 2001), since the color of HB stars depends very strongly on their age and metal content (see Fusi Pecci et al. 1993, for discussion and references). In particular, HB stars can be located to the blue of the RR Lyrae instability strip (Blue HB stars) only if they are very old (age $>10 \mathrm{Gyr}$ ) and (typically) metalpoor, while HB stars lying to the red of the instability strip (Red Clump stars) must be (comparatively) young or metal-rich, or both (see Monaco et al. 2003, and B06 for the interpretation of the HB morphology of Sgr in this context). Monaco et al. (2003) noted that both the BHB and RC sequences observed at the center of Sgr have a clear counterpart in the Color-Magnitude Diagrams (CMDs) of Stream fields obtained by N02 using data from the Early Data Release of the Sloan Digital Sky Survey (SDSS). Here we perform a direct comparison of the relative abundance of BHB and RC stars in the Sgr core and in one of the Stream fields studied by N02, to search for a population gradient. While the results we obtain are qualitatively similar to those of C06, they refer to a different and more distant portion of the Stream, and provide an on-field demonstration that the whole Sgr Stream can be studied with this technique.

\subsection{The data}

To obtain a representative sample of the stellar population in the core of Sgr we take the photometry of the $1^{\circ} \times 1^{\circ}$ wide field located $\sim 2^{\circ}$ eastward of the galaxy center, recently presented by B06, i.e. the $\operatorname{Sgr} 34$ field $\left((l, b) \simeq\left(6.5^{\circ},-16.5^{\circ}\right)\right.$, 


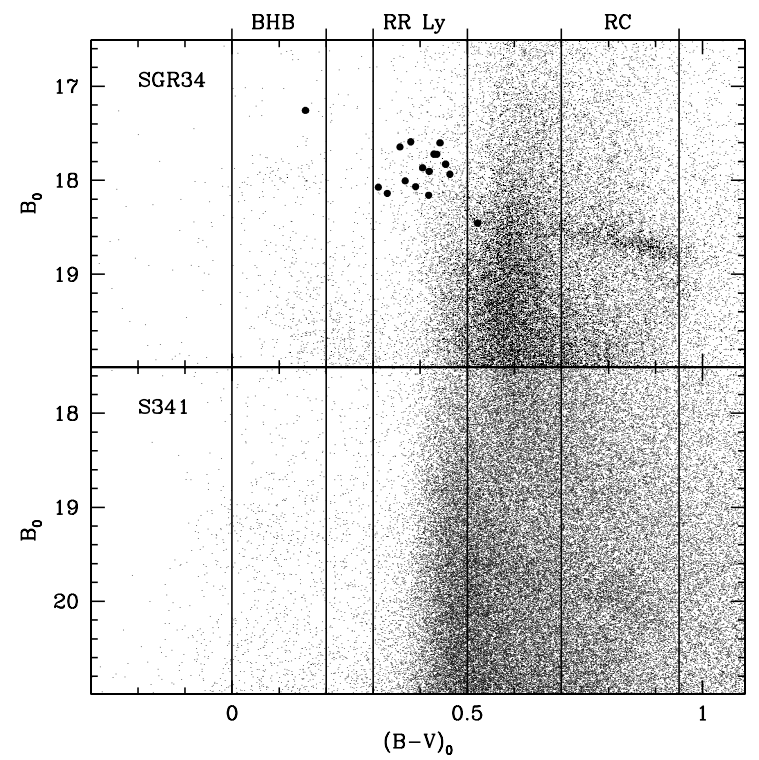

Fig. 1. CMDs focused on the Horizontal Branch for fields in the core of Sgr (upper panel) and in the Sgr Stream (lower panel). The color strips adopted to select candidate RC and BHB stars are enclosed within vertical lines and labeled. The filled circles in the CMD of SGR34 are the ab type RR Lyrae counter-identified in the nearby SGRW field from the catalogue of Cseresnjes (2001).

see B06 for details). N02 provided two very clear detections of the Sgr Stream in their fields S341+57-22.5 and S167-54-21.5, where the first two numbers of the names are the mean Galactic longitude and latitude. While the BHB is clearly visible in the CMD of S167-54-21.5, the signal of the Stream RC is too weak in this field to derive fully reliable star counts, due to various intrinsic factors that will be discussed elsewhere (Bellazzini et al., in preparation). Hence exclude this field from the analysis and we focus on S341+57-22.5 (S341 hereafter, for brevity), a $\sim 62.5 \mathrm{deg}^{2}$ strip enclosed within $200^{\circ} \leq \mathrm{RA} \leq$ $225^{\circ}$ and $-1.25^{\circ} \leq$ Dec $\leq 1.25^{\circ}$, that samples a portion of the leading arm of the Stream, lying more than $40 \mathrm{kpc}$ away from us in the Northern Galactic hemisphere (N02, Law et al. 2005). The $g^{*}, r^{*}$ photometry by N02 has been transformed into Jonhsons-Cousins $B, V$ according to Smith et al. (2002). The photometry has been corrected for extinction following B06. Note that the average reddening in the $\mathrm{S} 341$ field is $E(B-V)=$ $0.04 \mathrm{mag}$.

\section{HB star counts}

In Fig. 1 we present reddening-corrected $B, B-V$ CMDs focused on the HB of fields within the main body of Sgr and of the S341 field in the Sgr Stream. We chose the $B, B-V$ CMD because in this plane a larger section of the BHB sequence appears nearly horizontal, with respect to $V, B-V$ diagrams. This will allow a better background subtraction, according to the method that will be described below. Conversely, the $\mathrm{RC}$, that appears tilted in the CMDs of Fig. 1, is nearly perfectly horizontal in $V$, $B-V$ diagrams.

The filled circles superposed in the CMD of SGR34 are the ab type RR Lyrae variables we counter-identified in a field located $\sim 2^{\circ}$ westward of the center of Sgr (SGRW) from the catalogue of Sgr variables by Cseresnjes (2001). The wide area surveyed by Cseresnjes (2001) does not include the SGR34 field but has a large overlap with SGRW. The photometry is from the same survey of the SGR34 field, the data reduction has been performed as in B06. In our dataset the variables are observed at random phase: their color-magnitude distribution is useful here to indicate the position of the instability strip in our diagrams, that will help in the selection of relatively pure BHB and RC samples. The RC-labeled color strip $0.7 \leq(B-V)_{0} \leq 0.95$ encloses the Red Clump population in the main body (at $B_{0} \sim$ 18.7, upper and middle panel of Fig. 1) and in the S341 field (at $B_{0} \sim 20.0$, lower panel; the fainter mean magnitude with respect to SGR34 is due to a difference in distance between the populations sampled by the two fields, see N02 and Monaco et al. 2004). The BHB-labeled color strip $\left(0.0 \leq(B-V)_{0} \leq 0.2\right)$, on the other hand, encloses the more populated portion of the $\mathrm{BHB}$, far to the blue of the ab RR Lyrae, at $B_{0} \sim 18.0$ in SGR34 and at $B_{0} \sim 19.3$ in S341. Obviously there are BHB stars bluer than this limit; the adopted selection is optimally suited for the background subtraction technique described below. Since the comparison is purely differential this does not affect the results discussed below, but the adopted selection does not allow a complete census of BHB stars ${ }^{1}$. It can be seen from Fig. 1 that the $\mathrm{RC}$ in S341 is slightly bluer than in SGR34 (by $\simeq 0.05 \mathrm{mag}$ ). This may be interpreted as a signature of a difference in mean metallicity between the two populations. However, it cannot be excluded that relatively small color differences may be produced by less-than-perfect transformations between the $g^{*}, r^{*}$ and $B, V$, and/or by errors in reddening or calibration, while star counts should be essentially unaffected by these problems (see below).

While clearly visible in all the presented CMDs, both the $\mathrm{RC}$ and the BHB features are seriously affected by contamination from unrelated sources, either Sgr stars in different evolutionary phases $^{2}$ or foreground stars of our Galaxy. To remove the contribution of this generic "background signal" from starcounts we proceeded as follows: (a) we construct the Luminosity Function (LF) of the stars lying in a given color-strip (RC or BHB), (b) we fit the LF outside the peak associated with the $\mathrm{RC}$ and/or BHB features, (c) we subtract the fitted LF to the observed one, and (d) we sum the residuals within the narrow region enclosing the $\mathrm{RC}$ or BHB peak. A similar approach has been used before, see, for example, Harbeck et al. (2001). We adopt $B_{0}$ LFs for the BHB strip and $V_{0}$ LFs for the RC strip to obtain the cleanest signal as possible for the two features that greatly differ in color (temperature). The observed LFs are plotted in Fig. 2 for BHB stars, and in Fig. 3 for the RC stars. RC and BHB peaks are clearly visible in all the presented LFs, hence the adopted technique can be reliably used. The fact that the BHB and RC peaks in the S341 LFs are somewhat wider than those in SGR34 is probably due to an intrinsic difference in the distance profile along the line of sight of the two considered populations, the core of Sgr being more compact than the considered portion of the Stream. The results of the background-subtracted star counts are summarized in Table 1 . The square root of the total number of stars counted in the reported magnitude windows (i.e. before subtraction of the background), are adopted as errors on the star counts $\left(N_{\mathrm{BHB}}, N_{\mathrm{RC}}\right)$, according to Poisson's statistic. To check the effects of color shifts (due to metallicity gradients and/or errors in reddening or in the photometric calibration) we

1 This is why we find a smaller fraction of BHB stars relative to the sum of BHB and RC stars here than in Monaco et al. (2003). In that paper a more generous selection box was adopted, including also the vertical part of the BHB.

2 For example, most of the faintest stars in the Fig. 1 CMD within the BHB color strip belong to the Blue Plume population of Sgr (see B06). 


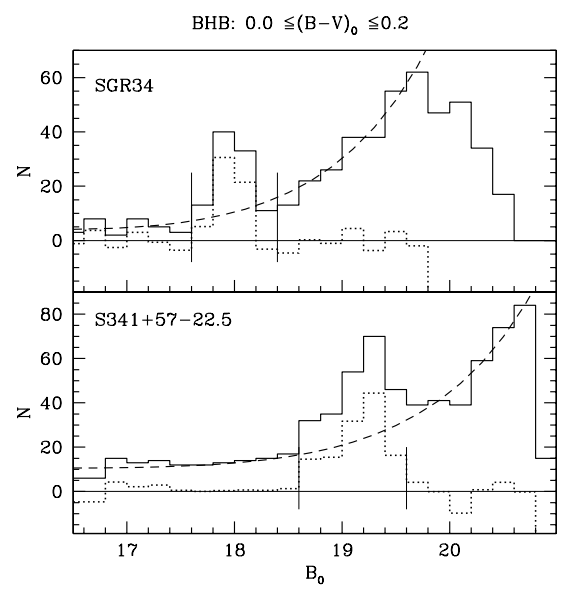

Fig. 2. LFs of the BHB color strip. The continuous-line histograms are the observed LFs, the long-dashed lines are the best-fit curves to the underlying "background" LF outside the BHB peaks, the dotted-line histograms are the residual of the observed $L F$ - fitted $L F$ subtraction. The thin vertical segments enclose the range in which we count BHB stars (the residuals of the fit) to obtain $N_{\mathrm{BHB}}$.

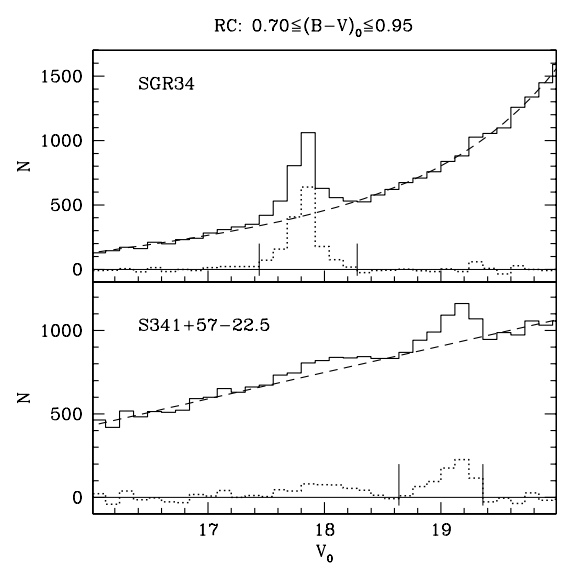

Fig. 3. LFs of the RC color strip. The symbols are the same as in Fig. 2. The thin vertical segments enclose the range in which we count RC stars.

repeated the whole procedure after shifting the selection strips by $\pm 0.03 \mathrm{mag}$. The resulting $N_{\mathrm{BHB}} / N_{\mathrm{RC}}$ ratios are unchanged, within the uncertainty, with respect to the values reported in Table 1. We also checked the case of a shift of -0.05 mag applied only to the RC strip of S341, to take into account the observed color shift of the Stream RGB in this field, and also in this case the change of $N_{\mathrm{BHB}} / N_{\mathrm{RC}}$ is smaller that the derived uncertainty.

Table 1 shows that the ratio of the number of BHB stars to the number of RC stars $\left(N_{\mathrm{BHB}} / N_{\mathrm{RC}}\right)$ is more than five times larger in the portion of the Stream sampled by the S341 (at $D_{\odot} \simeq 45 \mathrm{kpc}$ from us, and more than $50 \mathrm{kpc}$ away from the center of the parent galaxy) than within the core of the Sgr galaxy (at $D_{\odot} \simeq 26 \mathrm{kpc}$ from us). The detected difference between the two fields is significant at the $\gtrsim 4.8 \sigma$ level; given the hypothesis that the HB morphology in S341 is the same as in SGR34, we expect $N_{\mathrm{BHB}}=24 \pm 5$ in the Stream field, while we observe $N_{\text {BHB }}=122 \pm 15$. Hence, BHB stars are much more abundant in the Stream ( $15 \%$ of the whole $\mathrm{RC}+\mathrm{BHB}$ population) than in the main body of Sgr (just 3\%, with the adopted selection; see footnote 1). This is a strong indication that old and metal-poor stars are much more represented in the population that was stripped from Sgr in the past than in the presently bound core, providing
Table 1. Star counts in the BHB and RC.

\begin{tabular}{lcc}
\hline \hline & Sgr34 & S341+57-22.5 \\
\hline$N_{\mathrm{BHB}}$ & $54 \pm 10$ & $122 \pm 15$ \\
$N_{\mathrm{RC}}$ & $1542 \pm 67$ & $686 \pm 78$ \\
$\frac{N_{\mathrm{BHB}}}{N_{\mathrm{RC}}}$ & $0.035 \pm 0.007$ & $0.18 \pm 0.03$ \\
$\frac{N_{\mathrm{BHB}}}{N_{\mathrm{RC}}+N_{\mathrm{BHB}}}$ & 0.03 & 0.15 \\
\hline
\end{tabular}

an independent confirmation and extension of the results by M03 and $\mathrm{C} 06$.

The techniques adopted by M03 and C06 may be limited in their application by a low sensitivity (as the infrared colors of M-giants, M03) or by the faintness of targets in distant regions of the Stream (as the high-resolution spectroscopy of individual stars by C06). The approach adopted here cannot provide a direct probe of the metallicity distribution as done by C06, nevertheless it relies on a very sensitive diagnostic (i.e. the HB morphology) that can be successfully measured out to very large distances. Hence, it may give the opportunity to trace population gradients along the whole extension of the Sgr Stream. Note that the procedure described here and the data reported in Table 1 provide the basis (in particular the "Sgr core zero-point" of $N_{\mathrm{RC}} / N_{\mathrm{BHB}}$ ) to measure the population gradient in an homogeneous scale to anyone having access to CMDs of the Stream deep enough to sample the whole HB. This may even allow one to probe differences in stellar populations between wraps of the Stream lying in the same direction but at different distances, such as those seen by Belokurov et al. (2006) and modeled by Fellhauer et al. (2006).

It is apparent from Figs. 2 and 3 that the accurate location (in magnitude) of features like the RC and the BHB may provide powerful constraints on the distance and the structure of the Stream. We will discuss in detail these applications in a forthcoming contribution (Bellazzini et al., in preparation).

\section{Conclusions}

We have detected $\mathrm{a} \gtrsim 4.8 \sigma$ difference in the ratio of BHB to RC stars between the core of Sgr and a distant portion of its tidal Stream. BHB stars are 5 times more abundant - relative to RC stars - in the considered Stream field than within the main body of the galaxy, a clear signature of the presence of an age/metallicity gradient along the Sgr remnant.

C06 clearly states that the observed metallicity gradient within the Stream cannot be caused by "an intrinsic variation of the instantaneous mean metallicity of Sgr with time", because the chemical enrichment of Sgr was essentially completed much before than the Stream stars that display the gradient effect were stripped from the main body of their parent galaxy (see also B06). This leaves the presence of a radial metallicity/age gradient in the progenitor as the only viable explanation for the observed core/Stream gradient (note, however, that the simultaneous presence of stars stripped from Sgr in different periGalactic passages in the same portion of the Stream may also play a role; see Fellhauer et al. 2006). C06 notes also that the implied radial gradient should have been significantly stronger than that typically encountered in dwarf spheroidal galaxies. A comparison with the results by Harbeck et al. (2001) shows that in the galaxies of their sample the $N_{\mathrm{BHB}} / N_{\mathrm{RC}}$ ratio typically varies by a factor of $<3$, over the considered radial range, that is less than the factor of 5 we observe between SGR34 and S341. However changes of a factor of $\gtrsim 8$ are observed in Sculptor and 
Sextans, hence the value found here does not appear exceptional, although the exclusion of the hottest stars from our BHB selection may hide a larger difference.

According to the $N$-body models by Law et al. (2005) the stars populating the branch of the Stream sampled by the S341 field have been stripped during different orbital revolutions, in the past. Independently of the adopted model (adopting a spherical, oblate or prolate Milky Way halo), only $30 \%$ of the stars in this part of the Stream have been lost from the main body during the current peri-Galactic passage or during the immediately previous one: hence the observed population should be dominated by stars lost more than $\simeq 1.5-2$ Gyr ago (see also Belokurov et al. 2006 and Fellhauer et al. 2006). Thus the observed HB morphology should reflect the stellar mix in the outer regions of the Sgr progenitor as it was 2 to 4 peri-Galactic passages ago. We conclude that such a stellar mix was significantly more metalpoor, on average, than that observed in the present-day bound remnant.

Acknowledgements. This research is supported by the INAF-PRIN2005 grant CRA 1.06.08.02. H.J.N. acknowledge the support of NSF through the grant AST03-07571. The N-body models by Law et al. (2005) have been retrieved from the homepage of S. Majewski (http://www.astro.virginia.edu/ $\operatorname{srm} 4 \mathrm{n} / \mathrm{Sgr} /$ ). We are grateful to the referee (A. Aparicio) for his useful suggestions and comments.

\section{References}

Bellazzini, M., Ferraro, F. R., \& Ibata, R. 2003, AJ, 125, 188

Bellazzini, M., Correnti, M., Ferraro, F. R., Monaco, L., \& Montegriffo, P. 2006, A\&A, 446, L1 (B06)

Belokurov, V., Zucker, D. B., Evans, N. W., et al. 2006, ApJ, 642, L137

Chou, M.-Y., Majewski, S. R., Cunha, K., et al. 2006, ApJL, submitted (C06) [arXiv: astro-ph/0605101]

Cseresnjes, P. 2001, A\&A, 375, 909

Fellhauer, M., Belokurov, V., Ebans, N. W., et al. 2006, ApJL, in press [arXiv: astro-ph/0605026]

Fusi Pecci, F., Ferraro, F. R., Bellazzini, M., et al. 1993, AJ, 105, 1145

Harbeck, D., Grebel, E. K., Holtzman, J., et al. 2001, AJ, 122, 3092

Ibata, R. A., Irwin, M. J., \& Gilmore, G. 1994, Nature, 370, 194

Ibata, R. A., Lewis, G. F., Irwin, M., Totten, E., \& Quinn, T. 2001, ApJ, 551, 294

Law, D. R., Johnston, K. V., \& Majewski, S. R. 2005, ApJ, 619, 807

Majewski, S. R., Skrutskie, M. F., Weinberg, M. D., \& Ostheimer, J. C. 2003, ApJ, 599, 1082 (M03)

Martínez-Delgado, D., Gómez-Flechoso, M.Á., Aparicio, A., \& Carrera, R. 2004, ApJ, 601, 242

Monaco, L., Bellazzini, M., Ferraro, F. R., \& Pancino, E. 2003, ApJ, 597, L25

Monaco, L., Bellazzini, M., Ferraro, F. R., \& Pancino, E. 2004, MNRAS, 353, 874

Monaco, L., Bellazzini, M., Bonifacio, P., et al. 2005, A\&A, 441, 141

Newberg, H. J., Yanny, B., Rockosi, C., et al. 2002, ApJ, 569, 245 (N02)

Smith, J. A., Tucker, D. L., Kent, S., et al. 2002, AJ, 123, 2121

Vivas, A. K., Zinn, R., \& Gallart, C. 2005, AJ, 129, 189 